\title{
Bats and evolution
}

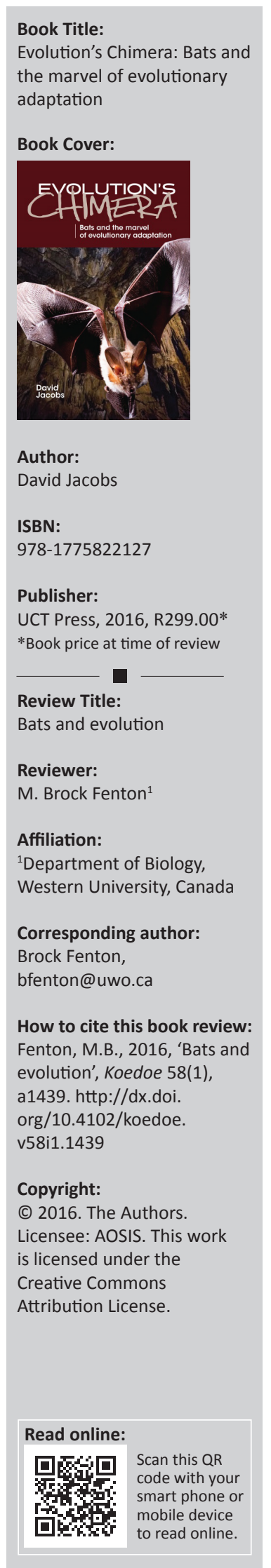

In this short book, David Jacobs uses bats to explore various aspects of evolution and evolutionary biology. In seven chapters, David goes from laying some ground work about evolution to considering how bats provide very good examples of evolution and evolutionary biology. Evolution and bats are the focus of the book, which David addresses to students of biology. The book is not a detailed or encyclopaedic review of bats, rather a series of specific examples of how he uses bats to illustrate the fundamental aspects of evolutionary biology. It is important to keep the focus in mind, even though some of the examples explored in detail do not involve bats.

In Chapter 2, classification and diversification of bats are explored, from body size to flight and echolocation. Chapter 3 deals mainly with the role that DNA analyses have played in extending our understanding of the evolutionary relationships among bats. Chapter 4 deals mainly with the possibility that competition (perhaps in the past) affected the diversification of bats. Chapter 5 focuses on sound-mediated interactions between bats and their insect prey. Chapter 6 considers the role that changes in echolocation signals might have played in bat evolution. Chapter 7 approaches bats and conservation and finishes with an appeal for bats and their conservation.

The book ends with a bibliography and an index, both of which are useful and appropriate. The book is well illustrated with a combination of drawings and photographs. Together, the illustrations support the main themes of the book.

Some readers will be puzzled about topics not covered in the book, specifically the importance of white-nose syndrome (WNS) and public health in the conservation of bats. WNS has had a catastrophic impact on populations of some bats in the United States and Canada where it is likely that some species of bats, including numerous common ones, will be extinct before too long. The role of bats in the spread of diseases that can afflict people (e.g. rabies, Ebola and SARS) may be real or imagined and surely strongly influence the public perception of bats.

It is 10 years since DNA barcode analysis was first used to document the details of bats' insect prey. Having this level of detailed information promises to revolutionise our view of bat ecology and behaviour. Furthermore, details of prey selection offer the opportunity to move from rhetoric about competition (past or present) to actual details of how trophic interactions affect (or do not affect) communities of bats. I did not find mention of this approach in the book.

I salute David for providing details of how bats can be used to advantage when studying evolution. Bat biologists will find his examples interesting, showing once again that each of us has her or his own favourite stories about bat biology and evolution. 